\title{
Serological Protein Profiling of Neuroblastoma by ProteinChip SELDI-TOF Technology
}

\author{
Qing-Yu He1,2, Rui Zhu³, Yi Ren³, Paul K.H. Tam³ and Jen-Fu Chiu'2,4 \\ ${ }^{1}$ Department of Chemistry, ${ }^{2}$ Open Laboratory of Chemical Biology of the Institute of \\ Molecular Technology for Drug Discovery and Synthesis, ${ }^{3}$ Department of Surgery, \\ ${ }^{4}$ Institute of Molecular Biology, University of Hong Kong, Hong Kong, China
}

Running title: Protein profiling of neuroblastoma

Correspondence to: Dr. Qing-Yu He, Department of Chemistry, University of Hong Kong, Pokfulam, Hong Kong, China. Tel: (852)2299-0787, Fax: (852)2817-1006, E-mail: qyhe@hku.hk

Abbreviations: SELDI, surface enhanced laser desorption ionization; MS, mass spectrometry; MW, molecular weight; pI, isoelectric point; PYY, peptide YY; PF4, platelet factor 4 precursor; PAO, polyamine oxidase; GSTM3, glutathione S-transferase isoform 3. 


\begin{abstract}
Serological proteins of neuroblastoma were profiled and analyzed by ProteinChipSELDI-TOF MS technology with five types of protein chips. By comparing with normal control, a number of protein or polypeptide signals were found significantly and consistently different in their intensities (expression levels) in tumor sera. Interestingly, 9 polypeptide peaks in these proteomic features can be simultaneously detected with consistent variations by more than one type of protein chips. None of the expression differences of these 9 polypeptides was found in similar comparisons between healthy controls and hepatomas. Preliminary protein identification showed hints for that some of these proteomic alterations may be closely related to the tumorigenesis of neuroblastoma. These results demonstrated the potential of serological biomarker identification for neuroblastoma by ProteinChip-SELDI technology.
\end{abstract}

Key words: neuroblastoma; tumor biomarkers; serum markers; protein profiling; SELDI; protein chips 
Neuroblastoma is a malignant solid tumor frequently occurring in childhood. This disease has an incidence rate of one case per 8000 children, accounting for $\sim 9 \%$ of all cancers in children under the age of 15 [Schwab et al., 2003]. Neuroblastoma arises from neuroectodermal cells derived from the neural crest and takes place in the adrenal medulla or paraspinal symphatetic nervous system [Schwab et al., 2003; Bown, 2001]. In general, infants less than one year old with neuroblastoma present with lower stage and less aggressive disease, which are usually chemosensitive and thus curable [Bown, 2001]. However, in children over one year old, the disease is often much more aggressive, metastatic and chemoresistant, and therefore has a high mortality rate [Bown, 2001]. The biological mechanism underlying this substantial clinical heterogeneity of neuroblastoma is still elusive.

To improve the understanding of neuroblastoma, many genetic and biological features have been investigated [Riley et al., 2004]. A number of studies have also been performed to identify tumor markers for facilitating the screening, diagnosis, prognosis, or monitoring of the disease to improve cure rates [Riley et al., 2004]. However, specific and sensitive diagnostic and prognostic markers have not been found yet. A recent study also revealed that relapse or progression in neuroblastoma cannot be reliably detected by monitoring the current tumor markers alone [Simon et al., 2003]. This presents a great need of continued effort to identify useful biomarkers for designing novel diagnostic tools and therapeutic strategies.

As an emerging powerful biological research technique, proteomics has recently become a key tool in the identification of biomarkers and disease targets [He et al., 2003; Zhu et al., 2003; Petricoin et al., 2003]. An advantage of proteomics is its ability to simultaneously inspect the whole proteome so that correlated proteins altered in expression and modification corresponding to a disease condition can be identified in a single experiment. This makes it 
possible to combine several protein markers together to form an index with higher sensitivity and specificity in the detection and monitoring of disease. 2D-gel based proteomics has been extensively used to study disease pathogenesis and to identify novel potential biomarkers for various cancers including neuroblastoma [Campostrini et al., 2004; Wimmer et al., 1996; Shin et al., 2003; Zhou et al., 2003]. However, 2D-gel electrophoresis has its limitation in protein separation. For example, proteins that have molecular weights (MW) less than $10 \mathrm{kDa}$ and isoelectric points $(\mathrm{p} I)$ less than 3 and higher than 11 cannot be separated or well displayed in gels. As a complementary tool, ProteinChip SELDI-TOF technology is much useful in analyzing proteins with low MWs covering the entire $\mathrm{p} I$ range. Other advantages of ProteinChip SELDI-TOF include its high sensitivity and straightforwardness especially in examining body fluids such as serum samples. In this study, serum specimens from neorublastoma patients were analyzed by using five types of protein chips. By comparing the serological protein profiles between patients and healthy controls, a number of proteomic features were found significantly altered. Some of the alterations can be detected in more than one type of protein chips simultaneously. None of these expression differences was found in similar comparisons between normal samples and hepatomas. The interesting results demonstrated that serological biomarker identification by ProteinChip SELDI TOF technology for neuroblastoma is promising. 


\section{METHODS AND EXPERIMENTAL PROCEDURES}

\section{Patient Materials}

With patients' consent, we collected blood samples from neuroblastoma patients at the Queen Mary Hospital, Hong Kong. The study was approved by the research ethics committee of the University of Hong Kong. Since few neuroblastoma cases were available, we only included 5 serum samples from patients admitted in Queen Mary Hospital in last two years. Table 1 lists the patients' information and histology. All neuroblastoma cases were confirmed histologically, with three at stage IV, one at stage III and one at stage II. Bone morrow metastasis was found at all the advance stages of IV and III. Serum samples from 10 healthy subjects were used as normal control. For comparison, another sample group containing 8 normal and 8 hepatoma sera was recruited and subjected to similar screening. All blood samples were distributed into $50 \mu 1$ aliquots and were stored at $-80{ }^{\circ} \mathrm{C}$ before assay.

\section{ProteinChip Analysis of Serum Samples}

Five types of protein chips including IMAC3, WCX2, SAX2, H50 and NP20 from Ciphergen Biosystems (Frement, CA, USA) were used in the protein profiling of the serum samples. These protein chips have different surface affinity properties with IMAC3 for metalbinding proteins, WCX2 (weak cation exchanger) for positive-charge molecules, SAX2 (strong anion exchanger) for negative charge molecules, H50 for hydrophobic peptides and NP20 (normal phase) for general binding of proteins. The IMAC-Cu metal binding chip was observed to give the most signals.

Each serum fraction was thawed and pretreated before loading. Serum sample of $3 \mu 1$ in an eppendorf tube was diluted into $6 \mu \mathrm{l}$ of U9 solution (9 mol/1 urea, 2\% CHAPS, 50 
mmol/l Tris-HCl, $\mathrm{pH} 9.0$ ), incubated for $30 \mathrm{~min}$, and mixed every $5 \mathrm{~min}$ in between when incubating on ice or shaken gently and continually in a cold room. After vortexing, each sample was diluted in 1:36 with corresponding binding buffers. Then $10 \mu 1$ mixed serum fraction was spotted onto each ProteinChip arrays with variety of chemical surfaces in duplicate.

Before spotting, IMAC-Cu ProteinChip was incubated with $10 \mu \mathrm{l}$ of $0.1 \mathrm{mmol} / \mathrm{l}$ $\mathrm{CuSO}_{4}$ solution for $10 \mathrm{~min}$ in a humid chamber. This was repeated twice. The chips were then incubated at room temperature for $30 \mathrm{~min}$ on a shaker. After incubation, non-bound proteins and other contaminants were removed by washing with buffers for three times. These buffers were $0.05 \%$ Triton $100+250 \mathrm{mmol} / 1 \mathrm{Nacl}$ in PBS for IMAC-Cu ProteinChip arrays, $50 \mathrm{mM}$ NaAOc (pH 4.0) for WCX2 ProteinChip arrays, $0.05 \%$ Triton 100 in $50 \mathrm{mmol} / \mathrm{l}$ Tris- $\mathrm{HCl}(\mathrm{pH}$ 8.0) for SAX2 ProteinChip arrays, $10 \%$ acetonitrile for $\mathrm{H} 50$ and $0.1 \%$ trifluoroacetic acid for NP20 ProteinChip arrays. Finally all chips were washed with MilliQ-deionized water for four times to remove interfering substances such as salts and detergents. On drying, $0.5 \mu l$ of saturated energy absorbing molecule solution [ $\alpha$-cyano-4-hydroxycinnamic acid in $50 \%$ acetonitrile $(\mathrm{v} / \mathrm{v}), 0.5 \%$ trifluoroacetic acid $(\mathrm{v} / \mathrm{v})]$ was added two times and the chips were allowed to air-dry.

\section{SELDI-TOF MS and Data Analysis}

Mass spectrometric analysis was performed by SELDI-TOF mass spectrometry in a PBS-II ProteinChip reader (Ciphergen Biosystems) according to an automated data collection protocol. This includes an average of 255 laser shots to each spot with a laser intensity of 240 and 265, respectively, dependent on the measured region (low $=2-20 \mathrm{kDa}$ and high $=20-200$ 
$\mathrm{kDa}$, respectively). The detector was run at a sensitivity of 9. Spectral analysis was carried out using the ProteinChip software version 3.1 (Ciphergen Biosystems). All mass spectra were normalized to have the same total ion current. Cluster analysis of the detected signals and the determination of respective $p$ values were carried out with the Biomarker Wizard Program (Version 3.0; Ciphergen Biosystems). For $p$ value calculation, spectra with at least 10 signals in the range between $2 \mathrm{kDa}$ and $20 \mathrm{kDa}$ exhibiting a signal-to-noise ratio of at least 5 were selected and analyzed with the Mann-Whitney $U$ test for non-parametric data sets. 


\section{RESULTS AND DISCUSSION}

In order to comprehensively analyze the serological proteins, five types of protein chips, H50, IMAC3, NP20, SAX2 and WCX2, were used for the protein profiling of the serum samples from neuroblastoma patients and healthy subjects. Figure 1 shows representative protein profiles from $1.5-20 \mathrm{kDa}$ detected by the five protein chips for the tumor and normal sera side by side. While the protein profiles among different chips are largely different, some proteomic signals can be visualized with more than one type of chips. Within each type of protein chip, significant differences in proteomic features between tumor and normal samples were evident.

When detailed cluster analyses were performed on the polypeptide peaks for each chip, a number of different features with a statistical significance were presented. Since only five cases of serum samples in tumor group were used in this study, we applied highly restricted parameters on selecting significant changes in protein expression. Only those polypeptide bands that varied more than 3 fold in intensity with $p$ values less than 0.05 were regarded as significant alterations. Under this condition, ten protein features showing significantly different in intensity between tumor and normal sera were detected by ProteinChip H50 array, with eight peaks obviously decreasing and the other two substantially increasing in expression in neuroblastoma (Table 2). Likewise, Table 3-6 list other proteomic features that show significantly differences between tumor and normal control detected by other four types of protein chips. Respectively, 17 peaks with 6 decreases and 11 increases in tumor were identified by IMAC 3 chip, 9 signals with 1 down and 8 up were visualized by NP20 chip, 8 features with 3 decreases and 5 increases were detected by SAX2 array, and 7 bands with 3 decreases and 4 increases were distinguished by WCX2 chip. 
Interestingly, careful comparisons among the proteomic variations detected by different protein chips revealed that some different peaks can be visualized consistently by more than one array. Figure 2 displays an expended area of protein profiles containing the protein bands that show similar trends of alterations with the five types of chips. Peak at 6934.3 Da cannot be detected by H50 array, but consistently shows enhanced intensities in tumor as measured by the other four protein chips. On the other hand, peak at 7767.6 Da can be seen by all five arrays and exhibits consistent decrease in expression in tumor sera. Other peaks that can be detected by more than one chip include those at 4058.65, 4215.34, 6919.62 , 8764.86, 9286.89, 13732.84, and $13868.63 \mathrm{Da}$, respectively. Table 7 lists all 9 protein features that can be identified by more than one array, in which two display constant increase and the other 7 were constantly depleted in tumor samples.

In a separated testing experiment, the positive control group containing 8 normal and 8 hepatoma serum specimens was also subjected to similar analyses by the 5 protein chip arrays. When cluster comparisons were made between normal and hepatoma samples respectively for each type of chips, many differences were observed in terms of expression intensity (data will be published elsewhere) but none of them resembles the similar trend of alterations with the 9 polypeptide features detected in the neuroblastoma training group. Only one peak at $8762.29 \mathrm{Da}$, which was detected by SAX2 chip and exhibited 3.1-fold decrease in intensity with a $p$ value of 0.011 , is close to but may not be the same to the peak at 8764.86 $\mathrm{Da}$ (see above). These results suggest that the proteomic features identified by the protein chips have a high specificity.

TagIdent tool in ExPASy molecular server (http://ca.expasy.org/tools/tagident.html) was used to search for a hint of the protein identities for these 9 polypeptide variants in 
neuroblastoma. All possible protein IDs in HOMO SAPIENS database are listed in Table 7. For example, the peak with a mass of 4058.65 Da matches to defensin 6 precursor with a molecular weight (MW) of 4058.65 Da, and peak of 4215.34 Da matches to peptide YY-like precursor (PYY) (MW: 4215.76 Da). Some of these matches can have more than one entry. For instant, peak of 8764.86 Da matches to apolipoprotein C-III precursor (MW: 8764.67 Da) and complement C4 precursor (MW: 8764.07 Da). Other possible matches can be found in Table 7 .

Although protein identification through TagIdent is basically preliminary, some proteins, if validated, are really related to tumorigenesis. For example, PYY is a regulatory neuropeptide that plays a significant role in inhibiting cancer cell growth [Tseng et al., 2002]. An under-expression of PYY has been implicated to the development and progression of colon adenocarcinoma and PYY treatment suppressed growth in pancreatic and breast tumors [Tseng and Liu, 2002]. PYY exerts action through its receptor [Freitag et al., 1995a]. Neuroblastoma cells have been shown to largely express PYY receptor [Kanatani et al., 1996; Freitag et al., 1995b; Miura et al., 1994], which may be a response to the less availability of PYY. The substantially decreased level of peptide YY-like precursor observed in current neuroblastoma sera may reflect the suppressed expression of the protein in cells, a relevant aspect in neuroblastoma development. Another low level polypeptide in neuroblastoma sera is platelet factor 4 precursor (PF4). PF4 is a CXC chemokine secreted by activated platelets and expressed during megakaryocytic differentiation. PF4 can inhibit endothelial cell proliferation in vitro and angiogenesis and tumor growth in vivo in experimental animals [Li et al., 2003; Long et al., 1990; Griffioen et al., 1999]. The marked decrease of PF4 level in cancer sera suggests the lacking activity of tumor inhibition of the protein in the patients. 
Two commonly and strikingly up-regulated proteins in neuroblastoma sera were identified as polyamine oxidase (PAO) splice variant 3 and glutathione S-transferase isoform 3 (GSTM3). PAO splice variant 3 is an isoform of the PAO enzymes [Murray-Stewart et al., 2002]. PAO takes part in the metabolism of polyamines, the organic cations with multiple functions in cell proliferation and differentiation [Thomas et al., 2003]. The regulation of this PAO enzyme has been implicated to have a role in facilitating apoptosis of cancer cells [Thomas and Thomas, 2003]. GSTM3 is a phenotype of the superfamily of GST enzymes involved in detoxification process. GSTs catalyze the glutathione conjugation reaction with eletrophilic compounds and carcinogens [Tsuchida et al., 1992]. The significant increase of GSTM3 level in neuroblastoma may correspond to the initiation of self protective machinery of cells in tumorigenetic process. The over-expressions of GSTs have been found in various cancers (For example [Lafuente et al., 1998; Li et al., 1997; Zhang et al., 1994]).

In summary, we have used five types of protein chips to examine the serum specimens from neuroblastoma patients and healthy subjects. Through SELDI-TOF mass spectroscopic analysis, a number of proteins or polypeptides were found to be significantly different in expression levels between the tumor and control sera. Some of these differences can be simultaneously distinguished by more than one type of protein chips, confirming that the alterations are stable and consistently detectable in serum. Possible protein identification revealed clues that these altered proteins may be related to the tumorigenesis of neuroblastoma. In addition, none of these alterations was found in the positive control group containing normal and hepatoma samples, indicating a high specificity for neuroblastoma. The present results demonstrate that ProteinChip SELDI-TOF technology is sensitive in identifying proteins/polypeptides changed in tumor serum and it is promising to combine 
these altered protein features as potential biomarkers for clinical application. More serum specimens should be screened to test the sensitivity and specificity of the proteomic features as diagnostic biomarkers.

\section{ACKNOWLEDGMENTS}

This work was partially supported by Hong Kong Research Grants Council Grants

HKU 7227/02M (to Q.Y.H.), HKU 7218/02M and HKU 7395/03M (to J.F.C.), the Department of Chemistry, and the Areas of Excellence scheme of Hong Kong University Grants Committee. 


\section{REFERENCES}

Bown N. 2001. Neuroblastoma tumour genetics: clinical and biological aspects. J Clin Pathol 54:897-910.

Campostrini N, Pascali J, Hamdan M, Astner H, Marimpietri D, Pastorino F, Ponzoni M, Righetti PG. 2004. Proteomic analysis of an orthotopic neuroblastoma xenograft animal model. J Chromatogr B Analyt Technol Biomed Life Sci 808:279-286.

Freitag C, Svendsen AB, Feldthus N, Lossl K, Sheikh SP. 1995a. Coupling of the human Y2 receptor for neuropeptide $\mathrm{Y}$ and peptide $\mathrm{YY}$ to guanine nucleotide inhibitory proteins in permeabilized SMS-KAN cells. J Neurochem 64:643-650.

Freitag C, Svendsen AB, Feldthus N, Lossl K, Sheikh SP. 1995b. Coupling of the human Y2 receptor for neuropeptide $\mathrm{Y}$ and peptide $\mathrm{YY}$ to guanine nucleotide inhibitory proteins in permeabilized SMS-KAN cells. J Neurochem 64:643-650.

Griffioen AW, Damen CA, Mayo KH, Barendsz-Janson AF, Martinotti S, Blijham GH, Groenewegen G. 1999. Angiogenesis inhibitors overcome tumor induced endothelial cell anergy. Int J Cancer 80:315-319.

He QY, Chiu JF. 2003. Proteomics in biomarker discovery and drug development. J Cell Biochem 89:868-886.

Kanatani A, Ishihara A, Asahi S, Tanaka T, Ozaki S, Ihara M. 1996. Potent neuropeptide Y Y1 receptor antagonist, 1229U91: blockade of neuropeptide Y-induced and physiological food intake. Endocrinology 137:3177-3182.

Lafuente A, Maristany M, Arias C, Cuchi A, Lafuente MJ, Molina R, Ballesta A, Trasserra J. 1998. Glutathione and glutathione S-transferases in human squamous cell carcinomas of the larynx and GSTM1 dependent risk. Anticancer Res 18:107-111.

Li TJ, Hirayama Y, Kitano M. 1997. Glutathione S-transferase pi-class as a tumour marker in lingual preneoplastic and neoplastic lesions of rats and humans. Virchows Arch 431:37-43.

Li Y, Jin Y, Chen H, Jie G, Tobelem G, Caen JP, Han ZC. 2003. Suppression of tumor growth by viral vector-mediated gene transfer of N-terminal truncated platelet factor 4 . Cancer Biother Radiopharm 18:829-840.

Long MW, Heffner CH, Williams JL, Peters C, Prochownik EV. 1990. Regulation of megakaryocyte phenotype in human erythroleukemia cells. J Clin Invest 85:1072-1084.

Miura M, Inui A, Sano K, Ueno N, Teranishi A, Hirosue Y, Nakajima M, Okita M, Togami J, Koshiya K, . 1994. Dynorphin binds to neuropeptide Y and peptide YY receptors in human neuroblastoma cell lines. Am J Physiol 267:E702-E709. 
Murray-Stewart T, Wang Y, Devereux W, Casero RA, Jr. 2002. Cloning and characterization of multiple human polyamine oxidase splice variants that code for isoenzymes with different biochemical characteristics. Biochem J 368:673-677.

Petricoin EF, Liotta LA. 2003. Clinical applications of proteomics. J Nutr 133:2476S-2484S.

Riley RD, Heney D, Jones DR, Sutton AJ, Lambert PC, Abrams KR, Young B, Wailoo AJ, Burchill SA. 2004. A systematic review of molecular and biological tumor markers in neuroblastoma. Clin Cancer Res 10:4-12.

Schwab M, Westermann F, Hero B, Berthold F. 2003. Neuroblastoma: biology and molecular and chromosomal pathology. Lancet Oncol 4:472-480.

Shin BK, Wang H, Yim AM, Le Naour F, Brichory F, Jang JH, Zhao R, Puravs E, Tra J, Michael CW, Misek DE, Hanash SM. 2003. Global profiling of the cell surface proteome of cancer cells uncovers an abundance of proteins with chaperone function. J Biol Chem 278:7607-7616.

Simon T, Hero B, Hunneman DH, Berthold F. 2003. Tumour markers are poor predictors for relapse or progression in neuroblastoma. Eur J Cancer 39:1899-1903.

Thomas T, Thomas TJ. 2003. Polyamine metabolism and cancer. J Cell Mol Med 7:113-126.

Tseng WW, Liu CD. 2002. Peptide YY and cancer: current findings and potential clinical applications. Peptides 23:389-395.

Tsuchida S, Sato K. 1992. Glutathione transferases and cancer. Crit Rev Biochem Mol Biol 27:337-384.

Wimmer K, Kuick R, Thoraval D, Hanash SM. 1996. Two-dimensional separations of the genome and proteome of neuroblastoma cells. Electrophoresis 17:1741-1751.

Zhang L, Xiao Y, Priddy R. 1994. Increase in placental glutathione S-transferase in human oral epithelial dysplastic lesions and squamous cell carcinomas. J Oral Pathol Med 23:7579.

Zhou B, Yang W, Ji JG, Ru BG. 2003. Differential protein expression induced by transient transfection of metallothionein-3 gene in SH-SY5Y neuroblastoma cell line. Sheng $\mathrm{Wu}$ Hua Xue Yu Sheng Wu Wu Li Xue Bao (Shanghai) 35:522-528.

Zhu H, Bilgin M, Snyder M. 2003. Proteomics. Annu Rev Biochem 72:783-812. 
Table 1. Patients' information and histology

\begin{tabular}{|c|c|c|c|c|}
\hline Patients & Age & Sex & Stage & Metastasis \\
\hline B370 & 2 & F & III & + \\
\hline B378 & 3 & M & IV & + \\
\hline B379 & 3 & M & IV & + \\
\hline B381 & 4 & M & II & - \\
\hline B405 & 6 & M & IV & + \\
\hline
\end{tabular}


Table 2. Proteomic features showing significantly differences in expression detected by ProteinChip H50 array.

\begin{tabular}{|c|c|c|c|c|c|c|}
\hline Mass (Da) & $\mathrm{p}$ & $\mathrm{Ctrl}(\mathrm{Mean})$ & $\mathrm{Ctrl}(\mathrm{SD})$ & Tumor (Mean) & Tumor (SD) & Fold \\
\hline 4215.79 & 0.0011 & 7.55 & 6.29 & 1.16 & 0.86 & -6.5 \\
\hline 4349.39 & 0.0011 & 17.69 & 3.79 & 6.35 & 0.92 & -3.0 \\
\hline 4389.11 & 0.0011 & 15.75 & 5.14 & 3.08 & 1.33 & -5.2 \\
\hline 4716.36 & 0.0011 & 32.95 & 5.74 & 9.67 & 6.42 & -3.4 \\
\hline 4818.34 & 0.0011 & 7.32 & 1.87 & 2.60 & 0.81 & -3.0 \\
\hline 4964.85 & 0.0067 & 4.85 & 2.31 & 1.55 & 0.94 & -3.1 \\
\hline 7569.77 & 0.0011 & 3.57 & 2.57 & 1.19 & 0.40 & -3.0 \\
\hline 7616.28 & 0.0048 & 1.57 & 0.43 & 7.73 & 6.01 & +4.9 \\
\hline 7766.53 & 0.0011 & 9.37 & 4.28 & 1.65 & 1.43 & -5.7 \\
\hline 8291.16 & 0.0048 & 0.71 & 0.27 & 3.33 & 3.69 & +4.7 \\
\hline
\end{tabular}


Table 3. Proteomic features showing significantly differences in expression detected by ProteinChip IMAC3 array.

\begin{tabular}{|c|c|c|c|c|c|c|}
\hline Mass (Da) & $\mathrm{p}$ & $\mathrm{Ctrl}(\mathrm{Mean})$ & $\mathrm{Ctrl}(\mathrm{SD})$ & Tumor (Mean) & Tumor (SD) & Fold \\
\hline 1236.05 & 0.0266 & 9.05 & 10.04 & 2.49 & 0.91 & -3.6 \\
\hline 1851.80 & 0.0351 & 1.17 & 1.53 & 8.15 & 7.80 & +7.0 \\
\hline 2025.87 & 0.0044 & 2.48 & 0.78 & 25.98 & 28.65 & +10.5 \\
\hline 2048.65 & 0.0114 & 1.29 & 1.06 & 7.11 & 6.45 & +5.6 \\
\hline 2063.75 & 0.0203 & 0.96 & 0.86 & 6.38 & 8.75 & +6.6 \\
\hline 2075.38 & 0.0452 & 2.86 & 1.48 & 9.61 & 8.13 & +3.4 \\
\hline 2794.13 & 0.0084 & 4.66 & 2.65 & 23.70 & 18.46 & +5.1 \\
\hline 3000.55 & 0.0203 & 1.57 & 1.12 & 5.51 & 5.48 & +3.5 \\
\hline 3434.51 & 0.0268 & 1.19 & 0.96 & 5.60 & 6.32 & +4.7 \\
\hline 4548.34 & 0.0153 & 5.03 & 2.02 & 30.32 & 18.62 & +6.0 \\
\hline 6434.81 & 0.0015 & 7.09 & 3.30 & 0.78 & 0.58 & -10.9 \\
\hline 6633.73 & 0.0016 & 18.28 & 11.68 & 3.13 & 1.76 & -5.8 \\
\hline 7566.69 & 0.0044 & 20.23 & 13.57 & 4.39 & 3.27 & -4.6 \\
\hline 7584.20 & 0.0268 & 11.17 & 11.55 & 2.03 & 1.04 & -5.5 \\
\hline 9085.31 & 0.0350 & 14.94 & 6.72 & 46.10 & 26.06 & +3.1 \\
\hline 10054.82 & 0.0350 & 0.69 & 0.53 & 3.13 & 3.29 & +4.5 \\
\hline 15116.16 & 0.0350 & 16.36 & 15.43 & 2.55 & 1.31 & -6.4 \\
\hline
\end{tabular}


Table 4. Proteomic features showing significantly differences in expression detected by ProteinChip NP20 array.

\begin{tabular}{|c|c|c|c|c|c|c|}
\hline Mass (Da) & $\mathrm{p}$ & $\mathrm{Ctrl}($ Mean) & $\mathrm{Ctrl}(\mathrm{SD})$ & Tumor (Mean) & Tumor (SD) & Fold \\
\hline 3375.66 & 0.0245 & 1.53 & 1.25 & 6.16 & 5.32 & +4.0 \\
\hline 4126.05 & 0.0027 & 2.09 & 1.81 & 6.23 & 2.85 & +3.0 \\
\hline 4158.17 & 0.0192 & 5.51 & 4.26 & 23.52 & 19.15 & +4.3 \\
\hline 4215.45 & 0.0246 & 15.12 & 9.02 & 4.78 & 6.29 & -3.2 \\
\hline 4471.27 & 0.0037 & 3.26 & 1.88 & 9.87 & 4.46 & +3.0 \\
\hline 4537.79 & 0.0148 & 0.83 & 0.62 & 7.00 & 12.20 & +8.5 \\
\hline 6923.62 & 0.0087 & 1.38 & 0.91 & 10.17 & 8.85 & +7.4 \\
\hline 6935.71 & 0.0066 & 1.41 & 1.27 & 15.15 & 11.47 & +10.7 \\
\hline 6946.09 & 0.0036 & 1.27 & 0.56 & 11.90 & 9.71 & +9.4 \\
\hline
\end{tabular}


Table 5. Proteomic features showing significantly differences in expression detected by ProteinChip SAX2 array.

\begin{tabular}{|c|c|c|c|c|c|c|}
\hline Mass (Da) & $\mathrm{p}$ & Ctrl (Mean) & Ctrl (SD) & Tumor (Mean) & Tumor (SD) & Fold \\
\hline 4058.63 & 0.0163 & 13.19 & 7.63 & 3.48 & 2.72 & -3.8 \\
\hline 4158.56 & 0.0039 & 7.97 & 3.33 & 34.06 & 27.21 & +4.3 \\
\hline 4214.58 & 0.0061 & 29.88 & 9.07 & 4.20 & 2.39 & -7.1 \\
\hline 4651.16 & 0.0065 & 1.24 & 0.29 & 12.19 & 18.22 & +9.8 \\
\hline 4780.32 & 0.0249 & 3.24 & 2.72 & 14.92 & 11.87 & +4.6 \\
\hline 5825.13 & 0.0373 & 1.54 & 0.82 & 4.34 & 2.60 & +3.0 \\
\hline 6919.49 & 0.0250 & 1.58 & 1.04 & 14.51 & 19.88 & +9.1 \\
\hline 12442.05 & 0.0163 & 2.34 & 1.22 & 0.71 & 0.54 & -3.3 \\
\hline
\end{tabular}


Table 6. Proteomic features showing significantly differences in expression detected by ProteinChip WCX2 array.

\begin{tabular}{|c|c|c|c|c|c|c|}
\hline Mass (Da) & $\mathrm{p}$ & $\mathrm{Ctrl}($ Mean) & $\mathrm{Ctrl}(\mathrm{SD})$ & Tumor (Mean) & Tumor (SD) & Fold \\
\hline 3978.25 & 0.0065 & 1.22 & 0.46 & 5.81 & 6.73 & +4.8 \\
\hline 4215.82 & 0.0062 & 23.85 & 8.16 & 3.97 & 6.27 & -6.0 \\
\hline 4271.01 & 0.0373 & 7.28 & 5.71 & 2.23 & 1.25 & -3.3 \\
\hline 6918.75 & 0.0249 & 3.25 & 1.09 & 15.60 & 13.77 & +4.8 \\
\hline 7766.43 & 0.0163 & 25.58 & 14.14 & 7.94 & 9.89 & -3.2 \\
\hline 11525.30 & 0.0249 & 0.64 & 0.33 & 10.66 & 18.76 & +16.7 \\
\hline 11987.32 & 0.0250 & 0.52 & 0.32 & 2.81 & 2.45 & +5.4 \\
\hline
\end{tabular}


Table 7. Fold differences ( $p$ values) for the polypeptide peaks detected co-currently by different types of ProteinChips.

\begin{tabular}{|c|c|c|c|c|c|c|}
\hline Mass (Da) & H50 & IMAC3 & NP20 & SAX2 & "WCX2 & Possible ID (Da) \\
\hline 4058.65 & & & $-2.6(0.061)$ & $-3.8(0.016)$ & & Defensin 6 precursor (4058.65) \\
\hline 4215.34 & $-6.5(0.001)$ & & $-3.2(0.024)$ & $-7.1(0.006)$ & $-6.0(0.006)$ & Peptide YY-like precursor (4215.76) \\
\hline 6919.62 & & & $+7.4(0.009)$ & $+9.1(0.025)$ & $+4.8(0.025)$ & Polyamine oxidase splice variant 3 (6916.88) \\
\hline 6934.29 & & $+2.8(0.045)$ & $+10.7(0.006)$ & $+4.0(0.054)$ & $+6.2(0.078)$ & GSTM3 protein (6933.16) \\
\hline 7767.63 & $-5.7(0.001)$ & $-2.4(0.020)$ & $-2.7(0.015)$ & $-2.3(0.011)$ & $-3.2(0.016)$ & Platelet factor 4 precursor (7769.18) \\
\hline 8764.86 & $-2.0(0.001)$ & & & $-2.3(0.025)$ & $-1.5(0.037)$ & $\begin{array}{l}\text { Apolipoprotein C-III precursor }(8764.67) \\
\text { Complement C4 precursor }(8764.07)\end{array}$ \\
\hline 9286.89 & & $-2.2(0.073)$ & $-2.4(0.015)$ & & $-2.1(0.037)$ & ZFM1 protein (9287.76) \\
\hline 13732.84 & & & & $-2.6(0.004)$ & $-1.9(0.037)$ & Tax interaction protein 1 (13734.71) \\
\hline 13868.63 & $-2.5(0.001)$ & $-2.7(0.045)$ & & $-2.6(0.016)$ & & $\begin{array}{l}\text { Myosin regulatory light polypeptide } 9 \text {, } \\
\text { isoform b (13865.55) } \\
\text { C1orf21 (13865.33) }\end{array}$ \\
\hline
\end{tabular}




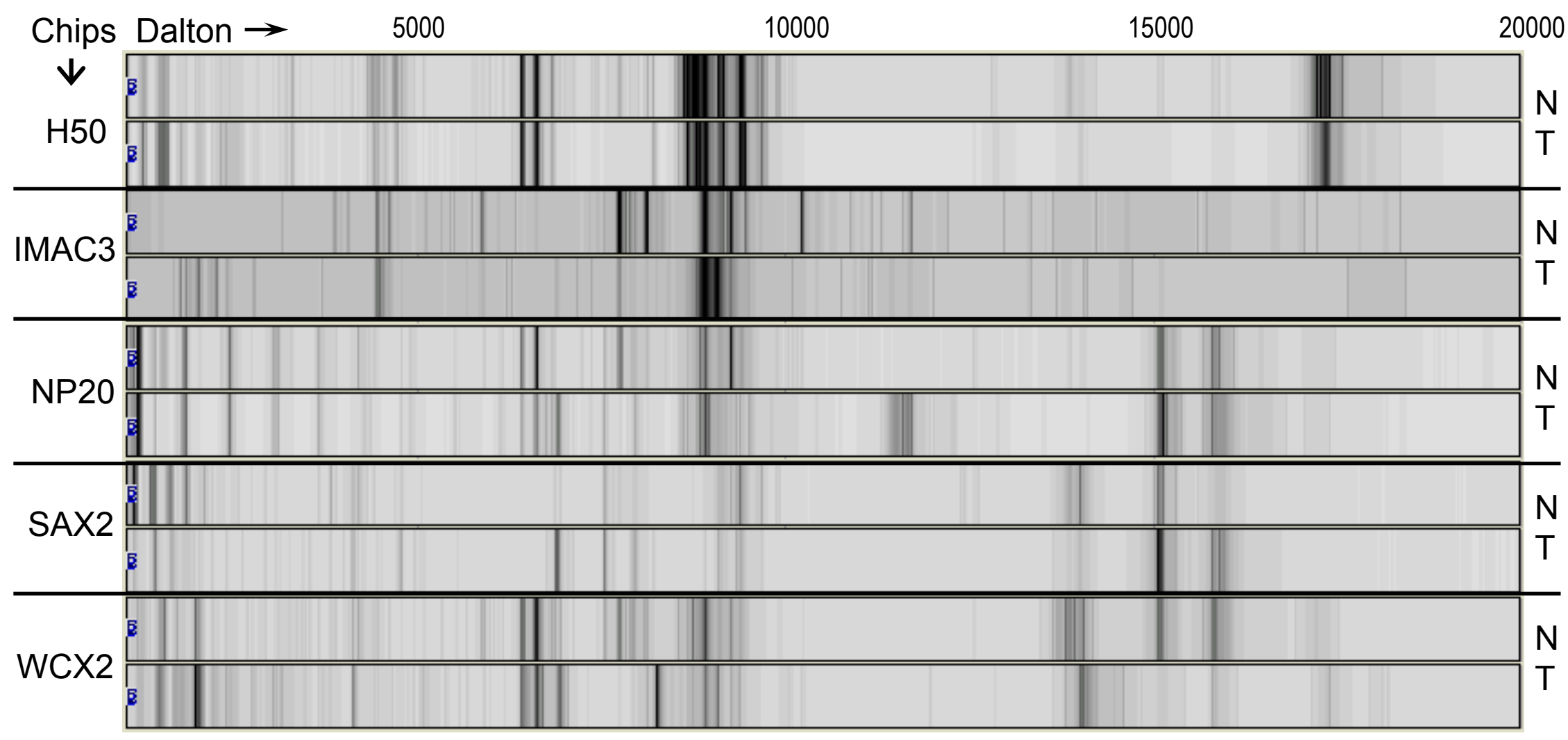

Figure 1. Representative protein profiles in $1.5-20 \mathrm{kDa}$ range detected by the five protein chips for the tumor and normal sera side by side. N: normal control, T: tumor. 


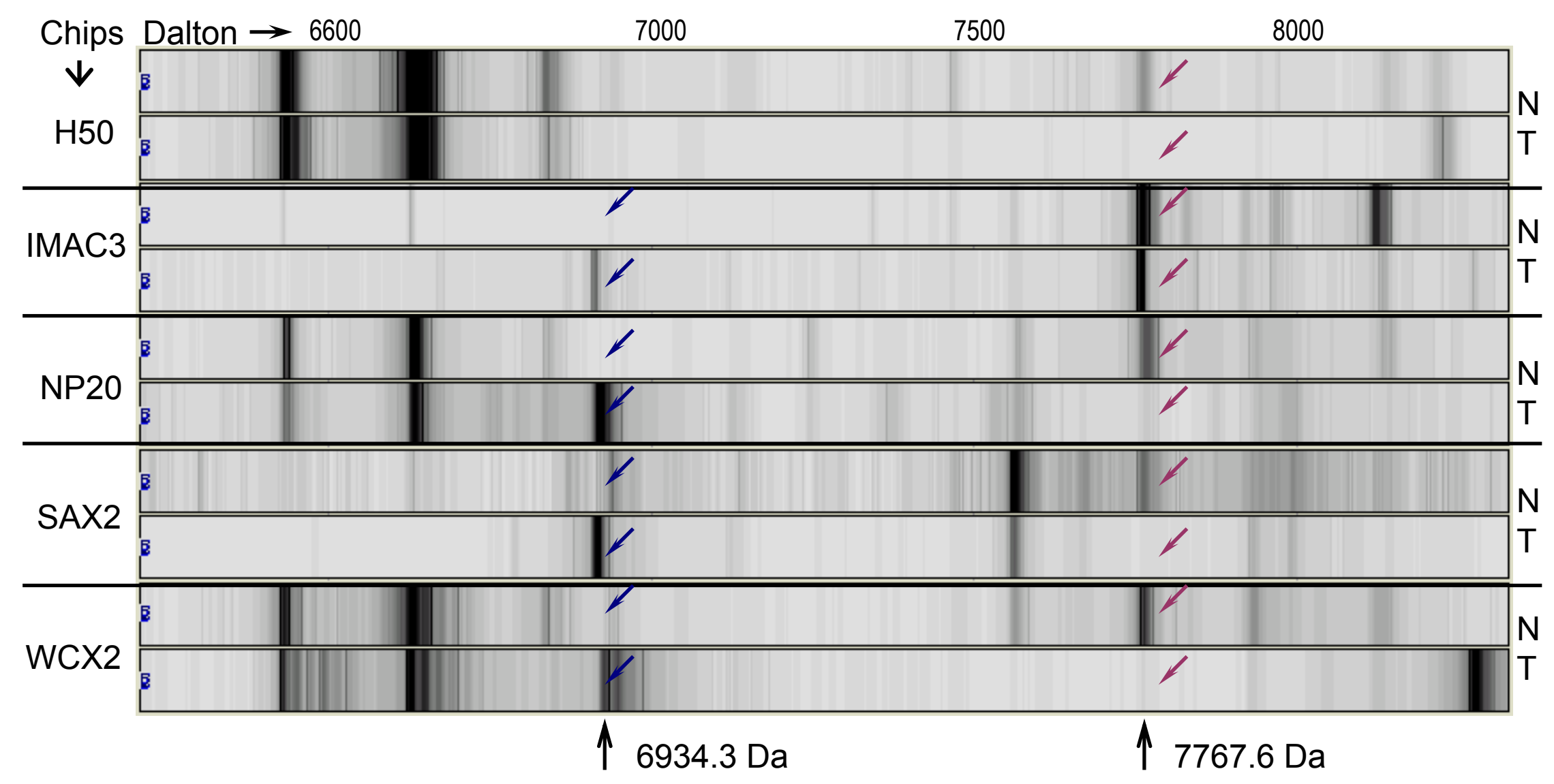

Figure 2. An expended area of protein profiles containing the protein bands that show similar trends of alterations with the five types of chips. N: normal control, T: tumor. 